Ali ebn e Abitaleb hospital in Rafsanjan, Iran. Data were collected during four months in 2000 . A total of 568 children with injuries were admitted to the emergency department. Data were analyzed using SPSS software.

Results: The ages ranged from 2 months to 10 years with a mean value of 6.5 years; $63 \%$ of patients were between 5 and 10 years of age. More males were affected than females, with a ratio of $1.7: 1$. Falls were the most common causal factor, responsible for 158 injuries (27.8\%); 120 (66.7\%) patients had fallen while on level ground while either playing or running; 55 patients $(9.7 \%)$ had fallen from a high altitude. Road traffic accidents were the mechanism of injury in 137 patients $(24.1 \%)$. About $86 \%$ of these were pedestrians knocked down by automobiles; vehicle passengers accounted for about $14 \%$ of the cases. Burns, mainly from scalding or dropping hot liquid, had occurred in 70 patients (12.3\%). There were 18 patients (3\%) with head trauma caused by a heavy object. Injuries from bites occurred in 7 patients (dog, cat, and bees). Drugs and toxin ingestion occurred in 6 patients (1.1\%), and sport injuries happened in 9 children. Other rare injuries were blade and knife wounds, toys, assault, and misplaced foreign bodies, or a complex of causes (15.8\%).

The most common anatomical regions affected were the head and limbs (73\%); 20\% had bony fractures; and 20.7\% with head injury, but only $20 \%$ of these injuries were severe. The home was the most common site of injury, followed by streets or roadway and school. Death occurred in 6 patients; accounting for $1.1 \%$; these patients had severe head injuries caused by motor vehicles.

Conclusion: The finding of this study suggest that falls and road traffic injuries are an important factor in childhood morbidity and mortality in our environment. These injuries are preventable and more parental care is advised. Keywords: children; injuries; locations; mechanisms; morbidity; mortality; parents; prevention; road traffic accidents; trauma Prebosp Disast Med 2002;17(s2):s88-s89.

\section{Rhabdomyolysis and Acute Renal Failure in Emergency ICU \\ Kazubisa Shimadzu}

Introduction: The mechanisms involved in rhabdomyolysis are not fully understood.

Method: The outcomes, the cause of death, complications, and treatment methods were reviewed for 64 patients with rhabdomyolysis cases and compared with those of 242 patients with acute renal failure (ARF) in an emergency intensive care unit (ICU).

Results: The mortality rate for the patients with rhabdomyolysis was $1.6 \%$, while that for the ARF cases was $35.5 \%$. Only one of the patients with rhabdomyolysis died due to acute respiratory failure. The complications accompanying ARF included: intracranial hypertension, adult respiratory distress syndrome (ARDS), circulatory instability, septicaemi, and disseminated intravascular coagulopathy (DIC). While multiple organ failure appeared as a complication in many of the ARF cases, it appeared in only a few of the rhabdomyolysis cases. Due to the presence of circulatory instability, continuous methods (continuous hemodialysis, continuous hemofiltration, and continuous hemo-diafiltration) were used in 116 of $142 \mathrm{ARF}$ cases (81.7\%) requiring blood purification. Of the 31 rhabdomyolysis cases requiring blood purification, 17 were treated successfully with conventional hemodialysis only. Aggressive fluid replacement during the early stage of acute renal failure accompanying rhabdomyolysis resulted in fewer cases progressing into chronic renal failure or death.

Conclusion: Rhabdomyolysis often is accompanied by renal disfunction. However, the effects are not as severe as are those with ARF. Moreover, the rhabdomyolysis cases developed fewer and less severe complications than did those with ARF. Rhabdomyolysis now is treated in this hospital with comparative ease.

Keywords: acute renal failure; chronic renal failure; complications; fluid administration; hemodiafiltration; hemodialysis; hemofiltration; mortality; purification; rhabdomyolysis

Prebosp Disast Med 2002;17(s2):s89.

\section{Web-Based and PDA-Based Chemical Hazard Query System for Chemical Incidents \\ $W e n-Y u Y u$}

Introduction: The aim of this study is to provide a quick query system for hazardous material of fixed facilities during a hazmat incident. The hazardous materials of fixed facilities in Taichung city were investigated.

Method: The characteristics and methods of handling the hazardous materials also were collected from a review of papers. A query system using the combination of the characteristics and where the hazardous material was spilled was built to decrease the numbers of possible hazardous materials. This database system was built up in either PDA-based or web-based system. The system has been tested during a disaster rehearsal.

Results: $76 \%$ of the users agreed that it is useful for enhancing the identification of hazardous materials.

Conclusion: To make the system more efficient, integrations of the database about hazardous materials in various government authorities must ensue.

Keywords: chemicals; database; disaster; hazardous materials; incident; query system

Prebosp Disast Med 2002;17(s2):s89

\section{Development of an International Emergency Nursing Program in Less Developed Countries Walter Jones}

Objectives: The provision of emergency nursing $(\mathrm{EN})$ is a growing need in less developed countries. Population growth, industrialization, increased motor vehicle use, traditional diseases, conflict, and increased vulnerability to events that may result in disasters create a growing need for emergency medicine care. Nurses play a key role in providing emergency care. An emergency nursing training course has been developed to build expertise in triage and primary and secondary assessment.

Methods: Emergency department nursing needs are defined by assessing the structure and resources of the emergency department and the educational background / $\mathrm{knowl}$ edge base of nurses. Based on the findings of the needs assessment, a nursing curriculum and course is developed with an evaluation component. This course and curriculum 
then are implemented using a train-the-trainers program. Once trained nursing leaders are prepared, they in turn will instruct student nurses in emergency department nursing. Results: This process has been put in effect in Gaza and the West Bank area of Israel. Training 24 trainers is under way, and these instructors will train 300 student nurses by 03 September. These nurses will be knowledgeable in the management of acute interventions in patients in the emergency department, using advanced triage methods, skills, and prioritization of patients' acuity with primary and secondary nursing assessment.

Conclusion: There is a rapidly growing need for trained nurses in emergency and disaster nursing internationally. Developing emergency nursing programs based on requirements determined by the needs of individual countries not works towards betterment of the healthcare delivery of that country. It should also help lower the overall mortality rate. Keywords: assessment; courses; emergency; healthcare; need; nurses; nursing; train-the-trainer, training

Prehosp Disast Med 2002;17(s2):s89-90. 